\title{
Activités
}

18-1 | 2021

Le programme de recherche cours d'action (2)

\section{Pratiques d'ergonomes et dynamiques de transformation dans le cadre de l'expertise SSCT pour les CSE}

Ergonomists' Practices when introducing changes in the context of

HSWC expertise

\section{Claire Violleau et Bernard Dugué}

\section{OpenEdition \\ Journals}

Édition électronique

URL : http://journals.openedition.org/activites/6048

DOI : 10.4000/activites.6048

ISSN : $1765-2723$

Éditeur

ARPACT - Association Recherches et Pratiques sur les ACTivités

\section{Référence électronique}

Claire Violleau et Bernard Dugué, "Pratiques d'ergonomes et dynamiques de transformation dans le cadre de l'expertise SSCT pour les CSE », Activités [En ligne], 18-1 | 2021, mis en ligne le 15 avril 2021, consulté le 17 avril 2021. URL : http://journals.openedition.org/activites/6048 ; DOI : https:// doi.org/10.4000/activites. 6048

Ce document a été généré automatiquement le 17 avril 2021

\section{cc) (†)}

Activités est mis à disposition selon les termes de la licence Creative Commons Attribution - Pas d'Utilisation Commerciale - Pas de Modification 4.0 International 


\title{
Pratiques d'ergonomes et
} dynamiques de transformation dans le cadre de l'expertise SSCT pour les CSE

\author{
Ergonomists' Practices when introducing changes in the context of \\ HSWC expertise
}

Claire Violleau et Bernard Dugué

\section{NOTE DE L'ÉDITEUR}

Article soumis le 6 avril 2020, accepté le 23 janvier 2021

\section{Introduction}

1 Depuis le $1^{\mathrm{er}}$ janvier 2020, les entreprises du secteur privé ont dû mettre en place une instance représentative du personnel unique : le Comité Social et Économique (CSE). Si certains éléments changent en profondeur (heures de délégation, formation des élus, budget, introduction du cofinancement sur les expertises dans le cadre de projet important, etc.), d'autres restent inchangés : le CSE se retrouve notamment avec les mêmes prérogatives en termes de santé, sécurité et conditions de travail que l'ancien CHSCT (Nivelet, \& Chevillard, 2019). Aussi, bien que cet article soit construit et basé sur des résultats d'expertises à l'époque des CHSCT, il éclairera le travail de l'ergonome, particulièrement au travers des notions de posture professionnelle et d'intelligence situationnelle dans le cadre de la réalisation d'expertises en Santé, Sécurité et Conditions de Travail (SSCT) ${ }^{1}$. 
2 Dans les multiples débats sur la pratique en ergonomie, l'expertise CHSCT a toujours particulièrement questionné les ergonomes (Etienne, \& Négroni, 1999). Si la fonction de l'expertise CHSCT a été souvent critiquée (Adam, \& Barnier, 2013; Bachelier, 2016; Cristafalo, 2009), la pratique de l'expert (qui n'est pas forcément un ergonome), sa manière de travailler et de se dégager des marges de manœuvre dans un cadre juridique contraint (Litim, \& Castejon, 2010), ont finalement été assez peu étudiées. Pourtant, cette forme de pratique révèle une dimension sociale, politique et stratégique importante par la nature du demandeur que sont les représentants du personnel au $\mathrm{CHSCT}^{2}$.

3 La loi régit le cadre des situations dans lesquelles le recours à un expert par les représentants des salariés au CHSCT peut s'exercer : dans le cas d'un risque grave ou pour tout projet important amené à modifier les conditions de travail (article L. 4614-12). Dans ce dernier cas, l'expert mandaté doit intervenir dans un délai de 30 à 45 jours et respecter une certaine méthodologie définie par l'arrêté du 23 décembre 2011. L'employeur peut contester certains éléments de l'expertise (durée, coût, modalités), mais ne peut s'y opposer sans un recours auprès du Tribunal de Grande Instance, et doit, de surcroît, la financer (à hauteur de $80 \%$ dans le cas des projets importants depuis la mise en place des CSE (L. 2315-94, L. 2312 84). Le recours à l'expertise est justifié par le manque de moyens ou de connaissances du CHSCT pour analyser un projet et ses répercussions possibles, pour être en mesure de rendre un avis motivé, ou afin de faire un diagnostic précis de situations de risque grave, souvent complexes, permettant d'élaborer des pistes de prévention pertinentes. L'expert est donc là pour aider les élus du personnel dans ce sens. Mais le recours à un expert correspond aussi à la volonté d'établir un rapport de force plus favorable vis-à-vis de la direction. En effet, il arrive fréquemment que la demande de recours à un expert soit la cristallisation de désaccords et de conflits dans le dialogue social (manque d'informations sur le projet, consultation tardive par rapport à son avancée, insuffisance d'analyse de ses conséquences sur les conditions de travail...). Pour toutes ces raisons, le cadre d'intervention de l'expertise CHSCT (L.4614-12, R4614-5-3) semble laisser peu de place à l'accompagnement et au déploiement de modifications des situations de travail sans sortir des contraintes légales, et en particulier des délais fixés. Cela amène souvent à penser que ces interventions sont majoritairement réduites à la compréhension du travail, à l'élaboration d'un diagnostic, plutôt que d'être orientées vers la transformation des situations de travail, ce qui serait le signe d'une pratique d'intervention ergonomique un peu «étriquée ", réduite à une posture "d'expert » et de « sachant».

4 L'intervenant étant un déterminant important de la réussite de l'intervention (Daniellou, 2006), nous avons souhaité explorer ce qu'il met réellement en œuvre, en supposant que son activité dépasse le registre de l'expertise externe définie par le Code du travail (la prescription), pour arriver parfois à engager de réelles transformations. Cela nous amène à nous interroger sur la nature même de ces transformations possibles.

5 Dans un contexte où d'un côté le métier d'ergonome se diversifie, et d'autre part les formes de la représentation du personnel connaissent une profonde transformation par la création du CSE et la suppression du CHSCT, il nous paraît important de chercher à comprendre les spécificités et les caractéristiques du travail de l'ergonome en expertise pour éclairer ce qui est mis en œuvre. L'investigation de cette problématique, au-delà 
de permettre une meilleure compréhension du travail de l'intervenant expert, ouvre aussi des portes au regard de la formation des ergonomes à ce type d'intervention, pour les aider à mieux appréhender les enjeux sociaux et politiques qu'elle revêt. Nous soutenons ainsi l'idée que l'expertise n'est certes pas une pratique spécifique de l'ergonomie puisque d'autres praticiens peuvent aussi la réaliser (sociologues, psychologues, anthropologues...), mais qu'il s'agit d'un contexte singulier dans lequel l'ergonome a une possibilité d'agir et d'intervenir tout aussi singulière, comme on peut d'ailleurs le dire pour d'autres formes de pratique en ergonomie, dans des cadres d'action très réglementés (les marchés publics par exemple), et dans des contextes où les résultats vont être utilisés de manière stratégique par les acteurs sociaux (Litim, Zittoun, \& Briec, 2012).

6 L'objectif de cet article est double: comprendre sur quoi et comment les transformations peuvent avoir lieu en expertise SSCT. Il s'agira donc de chercher à définir la nature des effets de l'expertise sur l'environnement socioculturel de l'entreprise et d'examiner les mécanismes, dans le travail de l'intervenant, qui contribuent à produire ces changements. Nous nous intéresserons alors particulièrement aux facultés d'adaptation, d'intelligence situationnelle de l'ergonome pour entrecroiser différents registres au cours de l'intervention et adopter alternativement des postures favorisant la production de transformations. Nous soutiendrons ainsi l'idée que les transformations sont rendues possibles grâce à la modulation et l'articulation de différents registres d'action.

\section{Intelligence situationnelle, registre et posture de l'intervenant}

7 Nous pouvons définir «l'intelligence situationnelle » ou «l'intelligence de situation » comme la capacité à comprendre une situation dans ses différentes dimensions et dans sa complexité pour s'y adapter et y apporter une réponse appropriée. C'est finalement "la capacité d'une personne à obtenir ce qu'elle souhaite et/ou à profiter des opportunités en ayant une bonne appréciation des personnes, de leurs attentes, des éléments bloquants et facilitants, des logiques d'action visibles et invisibles ainsi que des enjeux et des risques. » (Autissier, 2009, p. 19). Cette notion renvoie à celle d'action située définissant tout cours d'action comme dépendant, de façon essentielle, de circonstances matérielles et sociales (Schuman, 1987), et aboutissant à une codétermination entre l'action et la situation. Il existe plusieurs visions de l'action située, mais notre analyse se situera plus explicitement dans le cadre d'une approche interactionniste et écologique des situations de travail. Malgré des différences (Béguin, \& Clot, 2004), les points communs des deux approches résident dans l'idée que l'action est en partie déterminée (ou contrainte) par les cadres sociaux (le contexte, la prescription, l'environnement, les acteurs), mais en partie seulement. Les acteurs vont à la fois utiliser l'environnement comme une ressource (Kirsh, 1995), tout en cherchant à redéfinir le contexte d'action à travers leurs actions et interactions et à créer de nouvelles ressources nécessaires à leur propre action (Hammond, Converse, \& Grass, 1992). Ce sont donc les interactions qui régissent et structurent l'ordre social. Pour Hughes notamment (1996), l'activité des personnes s'inscrit dans une "matrice sociale " au sein de laquelle existent des "divergences de conception"sur ce que doit être leur travail. Les acteurs "n'acceptent pas complètement la définition du rôle qui leur est imposée 
d'en haut, mais élaborent leur propre définition en communiquant avec leurs pairs et en interagissant avec les populations qu'ils servent» (p. 68). Cette approche nous permet de mettre l'accent sur le fait que, dans la pratique de l'expertise et malgré un cadre légal très prescriptif, les acteurs ergonomes occuperont des "rôles" différents selon la manière dont les divergences sur l'action sont travaillées, et selon les marges de manœuvre leur permettant de mobiliser différents registres d'action, en s'appuyant sur les savoirs de métier. Dans cette optique, l'environnement contient à la fois des éléments auxquels il faut faire face et s'adapter, mais présente aussi des opportunités. Il revêt une forme non déterminée, produite par les interactions sociales, et peut aussi se construire et se préparer dans le sens de l'action que l'intervenant souhaite mener plus tard. Cela éclaire notamment la complexité que peut revêtir le " produit social » généré par l'intervenant.

8 Afin de mieux caractériser cette notion d'intelligence situationnelle dans le travail de l'intervenant, nous définirons les différents registres d'action qu'il porte sous le terme de «postures » catégorisées selon des « registres » distincts. Au sens décrit par Lameul (2008, p. 89) : «une posture est la manifestation (physique ou symbolique) d'un état mental. Façonnée par nos croyances et orientée par nos intentions, elle exerce une influence directrice et dynamique sur nos actions, leur donnant sens et justification ». Cette définition de la posture nous permet d'intégrer la dimension physique et psychique, mais aussi d'englober la vision interactionniste et écologique de l'action.

9 Pour apporter davantage de précision dans notre compréhension du travail de l'intervenant, nous tâcherons de catégoriser les différentes postures qu'il adopte selon la notion de registres pédagogiques (Dugué, Petit, \& Daniellou, 2010), traduisant et concourant à des objectifs différents :

- Le registre de l'expertise : où l'intervenant privilégie les connaissances et les compétences de sa spécialité et utilise principalement une méthode « expositive » ou démonstrative.

- Le registre de l'apprentissage : où l'intervenant cherche à construire du savoir dans et par l'action, à partir d'informations mises à disposition. L'intervenant va chercher à comprendre ce qui peut faire difficulté et se situer en accompagnant.

- Le registre de la relation : où l'intervenant ne cherche pas à transmettre explicitement des connaissances à ses interlocuteurs, mais tente de favoriser la découverte par eux-mêmes des principes qui font l'objet de l'apprentissage.

10 Dans la pratique, nous faisons l'hypothèse que les experts SSCT mobilisent ces différents registres selon les acteurs en présence et les contextes d'action. Ces arrangements singuliers seront différents d'un expert à l'autre, en fonction de l'expérience, des choix stratégiques, de la vision de leur rôle dans l'expertise.

11 L'intervention dans le cadre d'une "expertise CHSCT ", par sa terminologie, renvoie directement au registre de l'expertise. Cette sémantique peut ainsi fortement laisser entendre aux différents acteurs de l'instance qu'elle se traduira par un apport de connaissances sous un modèle d'échange plutôt « descendant » et démonstratif de la part de l'intervenant. Le rapport entre savoir et pouvoir est au cœur même du concept d'« expert» (Delmas, 2011), bien qu'originellement l'expert est celui qui porte l'expérience. Dans le langage usuel, et donc aussi dans le monde de l'entreprise, le terme «expert» sous-entend l'idée d'expertise unique et reconnue, l'expert étant perçu comme porteur d'une connaissance rare et précieuse, incontestable et indiscutable, bref l'expert est celui qui dit «vrai» (Boutte, 2007). Cette vision est d'autant plus renforcée par l'autorité de compétences de l'expert cautionnée par 
l'agrément ministériel ${ }^{3}$. Il est attendu de lui qu'il apporte des éléments d'analyse autour d'un diagnostic et d'un pronostic sur les conditions de travail, mais également qu'il présente des propositions d'actions et des pistes de solutions concrètes (arrêté du 23 décembre 2011).

Pour autant l'intervenant se cantonne-t-il à cette prescription ? Est-elle suffisante pour produire des transformations? Il nous semble que l'expertise offre la possibilité de transformer le système sociotechnique dans lequel elle se déroule, au sens où l'ont décrit Landry et Tran Van (2010), car les transformations ne revêtent pas uniquement une dimension physique, technique ou organisationnelle [sur les situations], mais peuvent également être d'ordre psychique, social ou culturel [sur les acteurs] (Guillon, 2002). Comme le propose Daniellou (1996), l'ergonomie est une pratique qui transforme les sujets (opérateurs, concepteurs, ergonome lui-même) avant de transformer les objets extérieurs. Dans cette perspective, une des finalités visées par l'expertise est (devrait être ?) le développement de la capacité des représentants des salariés à analyser les situations de travail, à élaborer des propositions d'amélioration et à se situer dans une perspective de prévention, autrement dit à développer le pouvoir d'agir $\mathrm{du}$ CHSCT. Une forme de contribution au développement du dialogue social.

Nous partirons de l'idée qu'une expertise CHSCT/CSE se situe toujours dans une tension, que l'intervenant cherche à travailler, entre l'alimentation d'un débat social qui prend parfois les allures d'un conflit, et la recherche de réponses à apporter pour qu'un projet prenne mieux en compte les caractéristiques du travail réel et les conditions de travail ; ou s'il s'agit de risques graves, qu'une démarche de prévention primaire soit mise en œuvre.

Nous proposons ici de traiter le sujet des transformations issues de l'intervention en expertise en apportant un éclairage sur les mécanismes "d'intelligence situationnelle » de l'intervenant se traduisant par une capacité, dans l'action, à saisir les enjeux, la complexité et à s'adapter aux circonstances pour apporter une réponse appropriée permettant d'atteindre les transformations visées.

\section{Matériel et méthodes}

\subsection{Les situations d'expertise analysées}

Pour appuyer notre propos, nous avons recueilli des éléments d'expertises portant sur des «projets importants ». Cela nous a notamment permis de mettre en lumière des éléments prépondérants de la conduite de projet autour de la notion d'intelligence situationnelle et de posture de l'intervenant. L'analyse du travail de l'ergonome dans ces missions d'expertise CHSCT s'inscrivait dans des contextes divers (Tableau 1) avec des dispositifs d'intervention spécifiques (Tableau 2). Il s'agit d'expertises réalisées au sein d'un grand cabinet agréé. Nous avons bien conscience que certaines caractéristiques sont spécifiques à ce cadre d'intervention, mais la plupart de nos réflexions nous semblent valables pour la pratique de l'expertise SSCT en général. 
Tableau 1 : Caractéristiques des missions étudiées. Table 1 : Characteristics of the missions studied

\begin{tabular}{|c|c|c|c|c|c|c|}
\hline $\begin{array}{l}\mathrm{N}^{\circ} \\
\text { expe } \\
\text { rtise }\end{array}$ & Entreprise & $\begin{array}{l}\text { Origine et sujet de } \\
\text { l'intervention }\end{array}$ & $\begin{array}{l}\text { Situation } \\
\text { syndicale }\end{array}$ & $\begin{array}{l}\text { Climat social } \\
\text { partenaires } \\
\text { sociaux }\end{array}$ & $\begin{array}{c}\mathrm{Nb} \text { de } \\
\text { personnes }\end{array}$ & Axes d'analyse conduits \\
\hline A & $\begin{array}{c}\text { Grande } \\
\text { distribution }\end{array}$ & $\begin{array}{l}\text { Transfert d'un magasin vieillissant } \\
\text { sur un site neuf; demande } \\
\text { d'expertise suite à un manque } \\
\text { d'informations et de réponses sur } \\
\text { le projet et sa mise en œuvre }\end{array}$ & $\begin{array}{l}4 \text { élus ; porté } \\
\text { par le DS FO } \\
\text { (syndicat } \\
\text { majoritaire) }\end{array}$ & $\begin{array}{l}\text { Très conflictuel; } \\
\text { crise entre le } \\
\text { secrétaire et le } \\
\text { président du } \\
\text { CHSCT }\end{array}$ & $\begin{array}{c}150 \\
\text { personnes } \\
\text { concernées }\end{array}$ & $\begin{array}{c}\text { Analyse des plans sous } \\
\text { l'angle des conditions de } \\
\text { travail actuelles et futures, } \\
\text { étude du plan } \\
\text { d'accompagnement salarial, } \\
\text { conduite du changement }\end{array}$ \\
\hline B & $\begin{array}{c}\text { Etablissement } \\
\text { bancaire }\end{array}$ & $\begin{array}{l}\text { Transformation des agences dans le } \\
\text { réseau : nouveau maillage, nouvelle } \\
\text { conception de l'accueil client } \\
\text { (horaires d'ouverture, services } \\
\text { proposés) visant des réductions de } \\
\text { postes et la mise en place de } \\
\text { polyvalence, remodelage des } \\
\text { différents postes (conseillers } \\
\text { professionnels et particuliers), } \\
\text { suppressions d'agences ; demande } \\
\text { d'expertise au vu des impacts du } \\
\text { projet et du nb de personnes } \\
\text { concernées }\end{array}$ & $\begin{array}{l}10 \text { élus ; union } \\
\text { intersyndicale }\end{array}$ & $\begin{array}{l}\text { Particulièrement } \\
\text { tendu à cause des } \\
\text { suppressions de } \\
\text { postes et la notion } \\
\text { de qualité du } \\
\text { service client }\end{array}$ & $\begin{array}{l}1840 \\
\text { personnes } \\
\text { concernées } \\
\text { dont } 166 \\
\text { suppressions } \\
\text { envisagées }\end{array}$ & $\begin{array}{l}\text { Analyse des situations } \\
\text { actuelles, changements } \\
\text { induits par le projet, enjeux } \\
\text { RH du projet, calendrier de } \\
\text { déploiement, enjeux du } \\
\text { projet sur les conditions de } \\
\text { travail, zoom sur les } \\
\text { " middle office " }\end{array}$ \\
\hline C & $\begin{array}{l}\text { Etablissement } \\
\text { bancaire }\end{array}$ & $\begin{array}{c}\text { Modification de la structure } \\
\text { hiérarchique avec suppression } \\
\text { d'une strate managériale (création } \\
\text { de ComDir transversaux) ; demande } \\
\text { d'expertise pour comprendre les } \\
\text { impacts sur les conditions de } \\
\text { travail, la charge de travail, la } \\
\text { gestion des carrières, le sens au } \\
\text { travail, les coopérations entre } \\
\text { réseaux, etc. }\end{array}$ & $\begin{array}{l}20 \text { élus ; union } \\
\text { intersyndicale } \\
\text { relative }\end{array}$ & Tendu & $\begin{array}{c}100 \\
\text { suppressions }\end{array}$ & $\begin{array}{l}\text { Impact du projet sur les } \\
\text { conditions de travail des } \\
\text { différentes filières } \\
\text { (entreprise, gestion privée, } \\
\text { retail), accompagnement du } \\
\text { projet et volet RH }\end{array}$ \\
\hline
\end{tabular}

$\mathrm{Au}$ sein du cabinet, les expertises sont triées en fonction de leur demande et des domaines d'expertise qu'elles requièrent, mais aussi des disponibilités des intervenants en mesure de les prendre en charge. En effet, le pilotage des missions est assuré uniquement par des consultants ayant le grade de "responsable de mission». Cette personne devient alors le "chef de file » et devra assurer le bon déroulement de l'expertise, ce qui comprend la relation client, mais aussi la constitution et l'animation de l'équipe d'intervenants qu'elle sollicitera pour répondre à la demande. Pour l'équipe d'intervenants, plus ou moins impliquée dans la construction de l'intervention, la prescription peut donc être double, provenant du client, mais aussi du chef de file, qui construit et définit, plus ou moins précisément, la réalisation de la mission (répartition des tâches, hypothèses et méthodes, thématiques de rédaction attendues, etc.).

Tableau 2 : Dispositifs d'intervention.

Table 2 : Modes of intervention

\begin{tabular}{|c|c|c|c|c|c|}
\hline $\begin{array}{c}\mathbf{N}^{\circ} \\
\text { expertise }\end{array}$ & $\begin{array}{c}\text { Nb de } \\
\text { consultants }\end{array}$ & Nature des compétences & $\begin{array}{c}\text { Nb de jours } \\
\text { vendus }\end{array}$ & $\begin{array}{c}\text { Délai } \\
\text { temporel }\end{array}$ & $\begin{array}{c}\text { Place de l'ergonomie } \\
\text { dans le projet }\end{array}$ \\
\hline $\mathbf{A}$ & 4 & $\begin{array}{c}\text { Ergonomes (2), sociologue, } \\
\text { ingénieur }\end{array}$ & 32 & 6 mois & $\begin{array}{c}\text { Majeure } \\
\text { (chef de file) }\end{array}$ \\
\hline $\mathbf{B}$ & 12 & $\begin{array}{c}\text { Ergonomes (2), anthropologue, } \\
\text { experts comptables et } \\
\text { financiers (4), spécialiste des } \\
\text { organisations, RH (4) }\end{array}$ & 121 & 1,5 mois & $\begin{array}{c}\text { Moyenne } \\
\text { (proposition de } \\
\text { méthodologie, analyses } \\
\text { terrain) }\end{array}$ \\
\hline C & 6 & $\begin{array}{c}\text { Ergonomes (3), RH, spécialiste } \\
\text { des organisations (2) }\end{array}$ & 45 & 2 mois & $\begin{array}{c}\text { Majeure } \\
\text { (chef de file) }\end{array}$ \\
\hline
\end{tabular}

\subsection{Méthodes employées}

17 Pour mener à bien cette étude, différentes méthodologies ont été empruntées à l'évaluation de la conduite de projet (Landry, 2008) : une méthodologie permettant de suivre le déroulé des trois interventions dans le but d'en tirer des éléments d'analyse 
communs, et une méthodologie permettant d'étudier le travail de l'ergonome au sein d'un cabinet, dans le contexte de l'expertise CHSCT.

\subsubsection{Le suivi du déroulé d'une intervention - analyse de la conduite de projet}

Pour comprendre l'intervention et ce qu'elle produit, l'ensemble des méthodes et des outils utilisés se sont inscrits dans une double visée: une visée spécifique (compréhension de l'intervention) et une visée généralisante (éléments transposables) permettant de faire des liens intra et inter-interventions. Ces outils sont des indicateurs de pilotage et de résultats (Tableau 3), basés sur les travaux de Landry et Tran Van (2010) ainsi que la constitution d'un journal de bord (Figure 1) (Bellemare, Marier, \& Allard, 2001). L'ensemble des éléments analysés provient de notes manuscrites, de verbalisations des intervenants et de l'étude des objets intermédiaires produits (comptes rendus de réunions, documents partagés/échangés, appels téléphoniques, mails...). Certaines données ont été retraitées a posteriori; la construction et le choix des éléments à relever s'étant construits et affinés au fur et à mesure.

Figure 1 : Trame utilisée du journal de bord dans sa version la plus élaborée. Figure 1 : Most elaborate version of the logbook used

\begin{tabular}{|c|c|c|c|c|c|c|c|c|c|c|c|}
\hline \multicolumn{5}{|c|}{ Evènement } & \multicolumn{3}{|c|}{$\begin{array}{c}\text { Informations factuelles sur le déroulé } \\
\text { de l'évènement }\end{array}$} & \multicolumn{3}{|c|}{ Eléments d'analyse = diag } & \multirow{2}{*}{$\begin{array}{l}\text { Commentaire } \\
\text { intervenant }\end{array}$} \\
\hline Date & Heure & Lieu & $\begin{array}{l}\text { Acteurs } \\
\text { présents }\end{array}$ & Type d'activité & $\begin{array}{c}\text { Objectifs } \\
\text { visés }\end{array}$ & $\begin{array}{l}\text { Résultats } \\
\text { obtenus }\end{array}$ & $\begin{array}{c}\text { Décisions } \\
\text { prises }\end{array}$ & Contexte & $\begin{array}{c}\text { Ensemble du } \\
\text { projet }\end{array}$ & $\begin{array}{c}\text { Suivi à } \\
\text { effectuer }\end{array}$ & \\
\hline
\end{tabular}

Tableau 3 : Les différents indicateurs pris en compte dans l'évaluation de la conduite de projet. Table3 : The different indicators taken into account in the evaluation of the project management

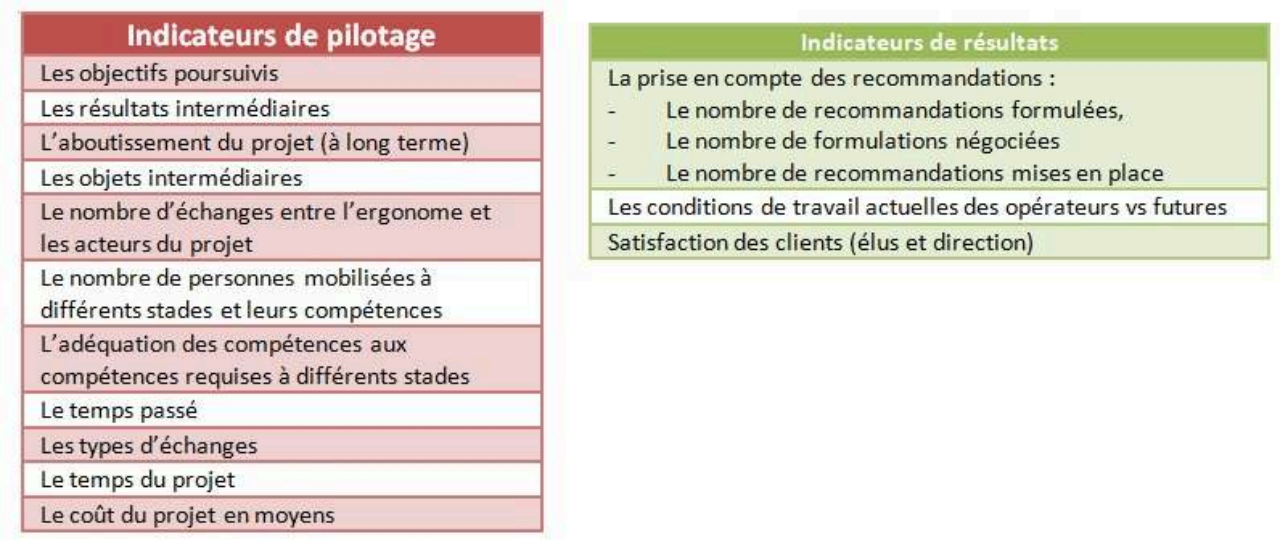

\subsubsection{L'analyse du travail de l'ergonome}

19 L'analyse de la pratique passe nécessairement, au préalable, par la compréhension du cadre d'exercice de l'intervenant. En effet, jamais complètement libre, son activité de travail s'inscrit dans des déterminants professionnels construits autour de contraintes temporelles, administratives, organisationnelles, etc. (Tran Van, 2010). Une fois le cadre cerné, différentes méthodologies ont été employées afin de chercher à capter les 
changements de posture de l'intervenant et de mettre en lumière son intelligence situationnelle :

- une analyse du travail de l'ergonome (Guérin, Laville, Daniellou, Duraffourg, Kerbuelen, 1997 ; Leplat, \& Cuny, 1974; Rabardel, Carlin, Chesnais, Lang, Le Joliff \& Pacal, 1998) où l'ergonome-chercheur est la même personne que l'ergonome-intervenant ;

- une analyse du travail d'ergonome par un autre ergonome participant aussi à l'intervention, mais plutôt en mesure de faire de la "gestion réflexive» au vu des spécificités de son positionnement dans l'équipe (ergonome junior en formation, mis en situation apprenante, et tutorée) (Petit, Querelle, \& Daniellou, 2007);

- un journal de bord (éléments pour pouvoir reconstituer un récit et en tirer des leçons);

- une prise de notes descriptives, méthodologiques et théoriques (Baribeau, 2004);

- des traces factuelles des différentes étapes (comptes rendus de réunions, entretiens...);

- une mise en débat avec un collectif partageant des normes, des règles de métier, des idéologies défensives de métiers ainsi que des savoirs formels socialisés (Samurçay, \& Rabardel, 2004).

Toutes ces méthodes font référence à la manière dont a été menée l'observation participante (Arborio, \& Fournier, 1999; Becker, 1985). Cette technique, issue de l'ethnographie et employée en sociologie, consiste à mener une analyse tout en étant intégré au processus de production, ce qui permet de développer une certaine "familiarisation" avec le milieu et d'obtenir des informations "les plus proches des faits». Elle est aujourd'hui reconnue comme outil utile et indispensable pour comprendre les systèmes et les organisations bien qu'elle ait fait débat au regard du retour sur soi, du relativisme et de l'esprit critique qu'elle nécessite pour atteindre ce niveau de réflexivité.

\section{Résultats}

Les résultats n'auront pas la prétention de mettre en lumière la totalité du travail de l'ergonome, mais seront focalisés sur les étapes de l'intervention qui nécessitent le plus de construction sociale, c'est-à-dire les phases d'interactions sociales où l'ergonome effectue un travail conjoint avec les acteurs de l'entreprise. Ces moments sont d'autant plus à forts enjeux pour la réussite de l'intervention qu'ils ne dépendent pas uniquement de l'intervenant ergonome et peuvent permettre la mise en place de transformations sociales, en particulier au niveau du dialogue social. Nous mettrons donc l'accent sur les pratiques en expertise qui sont porteuses de ces transformations.

\subsection{L'analyse de la demande et sa reformulation}

Matérialisée dans un premier temps par une rencontre avec les élus du CHSCT (demandeurs) et dans un second temps avec la direction (financeurs et décideurs), il n'est pas rare d'être confronté à des mécanismes de résistance de cette dernière. En effet, sa présence est parfois plus en lien avec l'obligation qui lui est faite que le signe d'une implication. Ainsi, à ce stade, pour (re)donner confiance aux acteurs, l'intervenant adopte différents registres évoqués plus haut :

- Celui de l'expertise pour apporter des connaissances (sur la méthodologie et des exemples de situations analogues) dans l'optique d'asseoir sa légitimité et montrer sa compréhension des préoccupations des acteurs. 
- Celui de l'apprentissage, particulièrement avec les élus pour coconstruire la proposition d'intervention. Dans l'expertise B, l'expert a sollicité la participation des élus pour les amener à s'approprier la démarche d'intervention, reformuler la demande et affiner le périmètre d'intervention.

- Celui de la relation, avec la direction, essentiellement, pour essayer de la faire évoluer dans ses représentations sur l'expertise CHSCT et, par "petites touches ", que cela instaure un lien de confiance (Corradi, 2015 ; Such, 2017). C'est le cas notamment de l'intervention A, où la direction, au départ dans une logique de contestation (pas de réponse, refus de donner des documents...) finira, après plusieurs échanges présentiels et téléphoniques avec l'expert, par admettre l'expertise. des autres par les nombreux échanges entre les élus et l'expert d'une part et entre l'expert et la direction d'autre part. Ils traduisent la recherche de compromis par l'intervenant, pour faire résonner les attentes du demandeur (les élus du CHSCT) avec certaines préoccupations de la direction afin de "l'embarquer", l'objectif étant d'instaurer un climat favorable à la construction de la démarche et au bon déroulement de l'expertise. L'ergonome intervenant, en cherchant à faire des liens entre enjeux de santé et de performance globale de l'entreprise (notamment de qualité des biens ou services produits) tient, en filigrane, un double objectif : celui du dialogue social et celui de l'objet de l'expertise en lui-même (le projet concerné).

À titre d'exemple, dans l'expertise A, l'opportunité d'intéresser les décideurs est passée par l'engagement du consultant à conduire l'expertise d'une manière à ce qu'elle puisse rétablir le dialogue social, véritable difficulté pour la direction dans la conduite de ses projets. Dans l'expertise C, embarquer la direction, c'était avant tout lui montrer que l'intervention pouvait lui permettre d'améliorer son projet en l'aidant à travailler sur le calibrage (actuel et futur) de la charge de travail des équipes.

Ces nombreux échanges sont également le reflet du besoin de connaître le contexte d'intervention (politique, économique, relationnel) et la spécificité de ses problématiques, informations que ne détient pas «l'expert». Il n'y alors ici pas de relation de «sachant - non sachant ", de " pouvoir et de savoir ». L'intervenant ne fait ici aucunement « autorité par sa personne ». En revanche, il peut influencer les choses en gagnant la confiance de ses interlocuteurs et en cherchant à se dégager des marges de manœuvre. Pour cela, il mobilise son expérience, la «bibliothèque de situations » qu'il s'est construite, et une capacité à recentrer les échanges sur les situations problèmes.

Ces capacités requièrent chez l'ergonome de l'expérience lui permettant de faire des analogies en temps réel, d'avoir une vision claire de ses objectifs (savoir où il va lui permet de réorienter les discussions) et une intelligence émotionnelle fine pour comprendre les défenses des acteurs et lever leurs craintes. Cela peut donc être particulièrement complexe pour un ergonome en formation ou junior. Dans tous les cas, une des ressources majeures développée par les intervenants observés est de se rendre à ce type de réunion (analyse de la demande et reformulation) à deux intervenants pour pouvoir se relayer, capter le plus d'éléments possibles sur l'instant et se construire par la suite une représentation partagée de la situation afin d'agir avec le plus d'efficience possible. 


\subsection{L'analyse du travail}

26 travail des salariés. En revanche, même s'il n'est pas strictement dans une posture d'expert, l'intervenant utilise son expertise sur le terrain au sens étymologique du terme puisque c'est son expérience qui lui permet d'assurer la qualité de l'intervention à travers sa connaissance de l'ingénierie des relations sociales, du fonctionnement du secteur d'activité et des process techniques et décisionnaires.

Le temps contraint est une caractéristique majeure de l'exercice. Ses compétences sont alors concentrées dans sa capacité à mener les entretiens et à tirer le substrat des problématiques très rapidement. Cette faculté s'exprime notamment par le fait que le consultant évolue dans sa manière de mener les entretiens.

Pour exemple, dans l'expertise A, lors de la réunion avec le chef de projet, "l'expert », qui a déjà découvert des premiers éléments sur des situations de travail par le biais d'observations de l'existant, se représente des hypothèses de travail qu'il teste et ajuste avec cet acteur. Ainsi, l'action dont l'objectif annoncé était de récolter des informations, se transforme en action stratégique puissante: familiariser l'acteur décisionnaire avec les résultats de l'expertise, l'inciter à réagir et à connaître son point de vue, enrichir l'analyse à travers la meilleure compréhension de sa logique et de ses contraintes, construire une relation basée sur l'échange et non la seule dénonciation.

Il s'agit ici de tout un travail pédagogique (registre de l'expertise et de la relation), au fil des différents échanges. L'expert sert de « guide » aux acteurs en les menant petit à petit vers une réflexion et un nouveau positionnement.

L'expertise $\mathrm{B}$ et $\mathrm{C}$ éclairent également la nécessité pour l'intervenant de tisser des relations sociales avec un interlocuteur du pôle RH qui puisse organiser et structurer l'intervention en interne d'un point de vue logistique. Véritable relais, particulièrement dans le cas d'interventions au périmètre large et dans les grandes entreprises, il permet un gain de temps en coordination, traduit l'implication de la direction et facilite la gestion des agendas des salariés. De plus, en fonction de la manière dont sont présentés l'intervention et l'intérêt d'y participer, mais aussi du lien qu'ont les salariés avec le relais RH, ils seront plus ou moins enclins à se rendre disponibles.

De fait, l'exemple opposé est celui de l'expertise A. Officiellement, la direction présente aux salariés l'expertise et son déroulement. Officieusement, elle fait passer le message aux salariés qu'il s'agit d'une démarche inutile et coûteuse, qu'y collaborer signifierait nécessairement la perte de leur prime de fin d'année. Cette tentative d'intimidation porte ses fruits puisque la journée balisée par l'intervenant pour recevoir des salariés volontaires en entretiens individuels se solde par un «silence radio» total. Pour contourner cette difficulté et pouvoir néanmoins rencontrer des salariés, l'intervenant doit alors imaginer, en temps réel, une autre modalité de rencontre. Il fera le choix ici de se rendre directement sur le «terrain » (puisqu'accessible) et de suivre des salariés en train de réaliser leur activité. Il faut alors reconstruire, pas à pas, et un à un, leur représentation de l'expertise (but, méthode, finalité) avant de pouvoir les interroger et accéder à leur vécu sur le travail et ses difficultés. C'est un enjeu fort pour l'ergonome de réussir à les rencontrer, car il joue son engagement auprès des élus qui l'ont mandaté et sa capacité de compréhension fine du réel (et donc de ses possibilités de transformations au sein de l'entreprise). 
nre de situations génère du stress supplémentaire pour l'intervenant et nécessite beaucoup de réactivité et d'inventivité de sa part pour dépasser les contraintes qui s’imposent à lui.

\subsection{La rédaction d'un rapport d'expertise}

Dans le cadre de l'expertise, il est attendu que l'expert mandaté réalise un rapport de son analyse et ce, dans plusieurs logiques : celle de répondre à la demande et de laisser une trace pour les élus et la Direction, mais aussi celle de produire un support sur lequel puisse se baser le Ministère pour évaluer la qualité du travail réalisé par l'expert agréé. Du fait des délais temporels courts et une charge importante d'écriture et de mise en forme, nous avons constaté que les ergonomes "expérimentés ", dès la phase d'observation, sont déjà en projection et en construction de leur rendu, ce qui peut être particulièrement difficile à acquérir pour un ergonome en formation.

31 L'étape de structuration du recueil de données se confond alors avec celle de l'analyse. De fait, quand ils sont sur le terrain, les intervenants expérimentés se questionnent en parallèle, en temps réel, sur la façon dont ils vont trier et ordonner les éléments et la façon dont ils vont rédiger. Ce classement anticipatoire des idées en cours de recueil de données leur permet de gagner du temps et d'être plus pertinents dans leur travail puisqu'ils sont en mesure d'ajuster en temps réel leurs futurs besoins d'informations. Ce processus donne également un aperçu de la charge mentale de l'intervenant ergonome et de sa capacité à poursuivre plusieurs buts en alliant différents raisonnements dans une même situation.

En nous basant sur l'analyse des rapports d'expertise, nous avons également constaté que leur construction est marquée par la volonté de les rendre pédagogiques et saisissables par les membres du CHSCT et est guidée, chaque fois, par trois niveaux de préoccupations et d'analyse : l'objet de l'expertise lui-même (ex. : le projet important), le mode de conduite du projet mis en œuvre par l'entreprise, et la pédagogie quant à la méthodologie utilisée par l'intervenant ergonome pour réaliser son analyse. Il est important de souligner, même si d'autres disciplines peuvent le prendre en considération, que ce triptyque est toujours fortement intégré dans l'approche de l'ergonome.

\subsection{La restitution d'un plan d'action aux acteurs}

Dans le cadre de la restitution du rapport d'expertise au CHSCT, l'ergonome guide ses actions dans le sens de favoriser les effets de l'intervention sur le milieu. Autrement dit, son objectif principal est de favoriser une approbation large de l'analyse proposée, voire des pistes d'action suggérées. Et ainsi faire en sorte que le rapport de l'expert soit soutenu, autant que possible, par les membres de l'entreprise qui devront continuer de le porter par la suite. Cela se traduit également par la volonté de changer les représentations des acteurs à travers un nouveau regard porté sur la situation analysée et la découverte de la richesse des données recueillies quand on porte une attention soutenue et méthodique sur le "travail réel». Mais il s'agit aussi d'outiller le CHSCT pour le rendre plus fort et en mesure de rendre un avis motivé sur les projets, sans se substituer à son action. Il est donc nécessaire pour l'ergonome d'éviter un effet de surprise lors de la réunion de restitution et de mener un travail préparatoire. 
Ces motivations se sont traduites dans les expertises A, B et C par des entretiens préalables et réguliers de l'intervenant avec les acteurs, l'intégration de ses préconisations sous forme d'hypothèses ou de questions au fur et à mesure des rencontres, une réunion préparatoire avec les demandeurs pour vérifier leur satisfaction, ainsi qu'une préparation minutieuse de la réunion de restitution (durée de présentation, nombre d'experts en présentation, positionnement dans la salle, etc.). Ces pratiques ont permis à l'ergonome d'ajuster son diagnostic pour gagner en pertinence, de valider et coconstruire les éléments de l'expertise et ainsi d'impliquer les acteurs. Cela a aussi permis d'améliorer ses conditions de travail dans le but de servir la qualité de la présentation (la réunion préparatoire est l'occasion d'anticiper et de calibrer la plénière).

Dans certains cas, ce travail peut se traduire par le fait de chercher à agir plutôt sur la méthode avec laquelle le projet a été conduit, en particulier lorsque des contraintes temporelles trop importantes bloquent la possibilité de modifier son contenu. Ce qui correspond à une adaptation de l'intervenant à son environnement et aux acteurs, et donc à une forme d'intelligence situationnelle.

Dans le cas évoqué, le «calibrage » de la réunion plénière a permis quant à lui de favoriser la stratégie de la présentation et d'assurer une gestion d'aléas, d'éviter les erreurs relationnelles/de figuration, c'est-à-dire de ne faire perdre la face à personne, ni à soi-même ni aux autres (Goffman, 1974), de crédibiliser le chef de file (vis-à-vis du coût généré par l'entreprise), et de confronter les logiques. L'ergonome en expertise CHSCT est ainsi en gestion permanente d'une tension entre la nécessité d'alimenter les élus pour renforcer leur position (le demandeur est bien la représentation du personnel au CHSCT), la volonté de construire un compromis avec la direction (qui est le décideur), et la nécessité des représentants du personnel de conserver une parole autonome, critique et objective (Dugué, Petit, \& Pinatel, 2012).

Dans le cadre de ces rencontres et points d'étapes multiples, nous avons constaté que l'intervenant change de registre en fonction des acteurs, des moments et de son interprétation subjective du moment.

Par exemple, l'enjeu de la réunion préparatoire de l'expertise A se situait avant tout dans la préparation de la réunion plénière du point de vue de la construction sociale. Il fallait réussir à faire comprendre aux élus qu'être dans une attitude d'offense (Goffman, 1959) ne favorisera pas la prise en compte de leur avis par la direction. L'apport de connaissances au cours de la réunion préparatoire n'était donc pas l'enjeu principal, car beaucoup d'éléments étaient déjà connus des élus. Le registre utilisé était alors plutôt celui de la relation avec une posture de " compagnon », le CHSCT abondant et complétant parfois les éléments apportés par les intervenants ; et celui de " guide " pour amener les élus à prendre conscience de la posture à adopter en réunion plénière.

Ces changements de posture de l'ergonome peuvent s'expliquer par le fait qu'il cherche avant tout à ce que l'ensemble des acteurs CHSCT puisse assumer la posture liée à leur fonction, et qu'ils réussissent à avancer de façon complémentaire et coordonnée sur les questions de santé et de sécurité des conditions de travail. C'est donc en cela que réside ses capacités de conseil : tantôt " compagnon », "guide ", " expert », il s'adapte pour rassurer, convaincre, objectiver et ainsi conduire les élus et la direction à un niveau de partage rendant possible les transformations.

Parfois, compte tenu de la consultation tardive du CHSCT sur le projet faisant l'objet de l'expertise, celui-ci est très avancé et les marges de manœuvre sont donc très faibles pour pouvoir en influencer le contenu. L'ergonome peut alors plutôt chercher à jouer «le coup suivant» (les futurs projets de changement), en laissant des traces de son 
travail, à la fois sur la manière d'analyser des situations de travail, de conduire des projets et d'en enrichir le contenu par une meilleure prise en compte du travail réel. Le rapport sera alors encore plus fortement marqué par un souci de pédagogie vis-à-vis des acteurs de l'entreprise.

\section{Analyse et discussion}

\subsection{Analyse de l'activité ou analyse du travail ?}

Nous pouvons nous interroger sur l'analyse qui est faite par l'ergonome en expertise. En effet, s'il réalise une analyse du travail, pouvons-nous dire pour autant qu'il réalise une analyse de l'activité au sens où la définit l'ergonomie?

Nous tiendrons le point de vue que l'ergonome, quelles que soient ses conditions d'exercice, regarde les situations de travail et les analyses avec une approche spécifique qui est celle de l'activité - sans pour autant faire systématiquement de l'analyse de l'activité. C'est ce qui le distingue des intervenants d'autres disciplines. Deux éléments nous semblent majeurs dans sa pratique pour le dire : l'écart entre le travail prescrit et le travail réel - qui suppose une attention particulière aux variabilités et aux régulations nécessaires pour y faire face - et la volonté de transformation.

Pour exemple dans l'expertise B dont l'objet est la transformation du réseau des agences, les spécificités des ergonomes, par rapport aux autres métiers représentés, ont été d'aller chercher la façon dont le travail se réalisait vraiment et pas uniquement la façon dont il était supposé se faire. En étant particulièrement attentifs aux variabilités, aux situations dégradées ou incidentelles, à la gestion des aléas, l'interrogation des salariés a porté sur la recherche de faits concrets illustrant leurs propos et les difficultés qu'ils rencontraient (récits d'expérience) dans des contextes de travail et d'interaction avec des clients précis.

41 Autrement dit, c'est la compréhension des régulations plus que celle des « conditions de travail » que l'ergonome est allé chercher, en quittant le registre de ce que «subissent " les agents pour aller vers celui de comment ils agissent, et même résistent, dans des contextes contraints, pour assurer un travail de qualité. Les ergonomes ont ainsi pu proposer des pistes de solutions pour trouver des compromis et des situations moins délétères du point de vue des conditions de travail et ainsi contribuer à la transformation de façon plus ou moins profonde du projet (configuration physique de l'accueil pour réduire les risques d'énervement des clients en voyant des conseillers censés être non disponibles, règles de fonctionnement de la polyvalence, charge de travail des conseillers et projection des tâches qu'il ne sera plus possible de faire avec la mise en place de la polyvalence à l'accueil, etc.).

Le cas de l'expertise B permet également d'aborder la question de la relation de l'ergonome avec des cointervenants d'autres disciplines. Plus l'équipe est de taille importante et diversifiée et plus elle nécessite des cadrages préliminaires et en cours d'intervention pour s'assurer de la construction de positionnements communs et ne pas tomber dans la juxtaposition de postures et de méthodologies. Cela amène parfois l'ergonome à redéfinir les concepts théoriques de sa discipline pour nourrir les discussions sur les méthodologies à déployer dans l'intervention et la façon de regarder une situation. 


\subsection{La démarche générale d'intervention et ses spécificités}

Finalement, le premier constat est que l'expertise CHSCT suit les mêmes étapes d'intervention que celles décrites par Guérin et al. (1997), avec cependant quelques particularités liées au contexte et à la difficulté d'évaluer les transformations initiées.

- L'analyse de la demande apparaît comme une phase de construction sociale, tant pour faire accepter l'expertise à la direction que pour faire une analyse stratégique pour la suite de l'intervention. Elle est également le moment de se dégager des marges de manœuvre et d'intervention acceptables si la direction maintient une position de rejet. Cette étape permet de comprendre le contexte, l'historique de l'entreprise et de l'émergence de la demande, la situation et le positionnement des différents acteurs ainsi que les enjeux de la demande (politiques, sociaux, économiques et temporels). Elle se solde par la mise en place d'une proposition d'intervention où la méthodologie de construction est similaire à celle d'une intervention plus «classique » (définition d'un périmètre, négociation d'un budget temps, mise en accord sur la méthodologie...). Encore une fois, l'ergonome n'est pas ici dans une démarche descendante prescriptive et dominante, mais plutôt dans le registre de la relation où l'objectif est avant tout de trouver des compromis et des solutions de mise en œuvre. Il joue un rôle d'intermédiaire et d'accompagnateur pour amener les acteurs à un accord permettant le lancement de l'intervention dans des conditions favorables et limitant au maximum les tensions.

- L'analyse du travail. L'essentiel des méthodologies est basé sur des observations, des entretiens et de l'analyse documentaire. Une des conditions majeures d'intervention pour les ergonomes est bien de pouvoir accéder au terrain. L'expertise se différencie néanmoins par son degré d'analyse du travail, n'allant pas toujours au cœur de ce qui se joue dans la complexité de l'activité réelle (en partie pour des raisons de délais, financières ou logistiques). Pour autant, selon l'objet de l'expertise, l'analyse fine de l'activité n'est pas toujours nécessaire pour porter une analyse et un regard critiques sur les situations en question et sur le projet lui-même. Comme dans toute intervention, l'ergonome cherche avant tout la pertinence et l'efficience, c'est-à-dire l'adaptation de « la maille » d'analyse au sujet traité. Selon nous, une des principales spécificités de son travail est de savoir tirer, en simultané de ses lectures et de ses rencontres avec les acteurs, des hypothèses pour (ré)orienter très rapidement son analyse et ses préconisations.

- La restitution aux acteurs est sans doute une étape qui se différencie d'une intervention « classique » par la forme qu'elle revêt, son caractère stratégique, plus que par son contenu (remise d'un rapport formalisé, réunion préparée avec un ordre du jour). C'est une étape particulièrement complexe en expertise, car beaucoup de choses non prévues peuvent se produire. Il faut être vigilant en permanence sur la façon dont les acteurs reçoivent l'information et la mettent en débat dans l'instance. En effet, il arrive que la stratégie mise en place par l'expert pour favoriser une prise de position par la direction sur un sujet et dans l'intérêt des élus soit contrecarrée, inconsciemment, par un représentant du personnel qui désoriente le cœur du débat en se fixant sur un seul élément symptomatique du problème à traiter. Pour exemple dans l'expertise $\mathrm{B}$, l'annihilation d'un débat sur la remise en cause de l'organisation future (notamment dans le dimensionnement des effectifs) en le focalisant sur un problème actuel et récurrent de remplacement. Si la stratégie de l'expert était d'amener la direction à reconsidérer à la baisse le nombre de suppressions de postes, l'élu détourne inconsciemment le sujet et offre une échappatoire à la direction. Mais il existe aussi le cas de figure opposé où la direction maintient une posture de « combat ».

La restitution aux acteurs comprend également une dimension pédagogique forte avec un 
objectif spécifique d'accroissement de la capacité des représentants du personnel à agir dans le cadre de leur mandat. De fait, en tant qu'expert, il ne s'agit pas seulement d'analyser le problème (risque grave ou projet important), mais aussi d'amener les représentants du personnel à travailler différemment, à porter un regard sur la manière dont les projets sont conduits, dont la prévention est organisée, etc., et ouvre finalement sur la question de la formation des élus.

Le recours à l'expertise a pour objectif premier de conseiller les représentants du personnel et de les accompagner dans leurs tâches d'analyse pour qu'ils soient en mesure de saisir les sujets importants de l'entreprise en cours ou à venir, et de peser sur les choix et orientations de l'employeur. De fait, certaines représentations de l'expertise CHSCT pourraient nous pousser à la considérer comme moins propice ou favorable aux transformations immatérielles (façon de penser et d'agir des acteurs) et matérielles, à cause des délais temporels, des conditions d'intervention, de la perception d'un manquement au niveau de l'accompagnement, etc. Les exemples présentés précédemment montrent qu'il est pourtant tout à fait possible d'en faire un outil de transformation: implication des acteurs dans l'enrichissement du diagnostic, engagement de l'instance dans la prévention, rétablissement du dialogue social le temps du projet, appropriation des préconisations de l'expert par les élus et capacité à les mettre en débat, légitimité des préoccupations de l'instance aux yeux de la direction... L'expertise peut donc être un moyen de réguler des tensions, de ne pas escamoter la dynamique positive du conflit, tout en cherchant à construire des compromis et des points de convergence. Il s'agit au bout du compte d'aider les membres du CHSCT à travailler les tensions qui traversent leur activité (Dugué, \& Petit, 2018), comme condition au développement de leur capacité d'action. Autrement dit, l'objectif est de faire des représentants du personnel des acteurs à part entière dans le champ de la santé et sécurité au travail.

Nous pouvons aussi considérer que cette forme de pratique de l'ergonomie souffre, avant tout, d'un manque de visibilité entre «l'état initial» et «l'état après transformations »- qui ne peut cependant s'apprécier que sur le temps long (Teiger, 2008) - en particulier parce qu'elle est marquée par les spécificités liées aux conditions d'intervention :

- Les délais temporels contraints rendant difficile la mise en place "d'indicateurs de réussite» avec les acteurs. La seule visibilité du résultat se situe alors dans le moment d'échange avec les élus en fin de réunion (intermédiaire et plénière) pour les faire s'exprimer sur leurs ressentis et plus largement sur la façon dont a été menée l'intervention et ce qu'elle a produit. Parfois, des contacts ultérieurs pourront avoir lieu avec les acteurs, mais ceux-ci sont loin d'être systématiques.

- Le moment de sollicitation de l'intervenant et ce qu'on attend de lui par rapport au cours du projet. En effet, il est appelé pour aider les élus à émettre un avis, du point de vue de la SSCT, sur un projet qui n'est pas encore mis en œuvre. Même si l'on peut déplorer qu'il n'y ait souvent pas d'association du CHSCT au projet (sous diverses formes possibles), ni de prise en compte du travail dans les choix structurants, nous pouvons valoriser le fait d'être en mesure de le faire évoluer avant qu'il ne se concrétise. La force de l'expertise pour faire bouger les lignes semble alors se situer d'un côté dans le rappel à la loi (l'employeur étant responsable pénalement de la santé et sécurité de ses salariés avec une obligation de résultat) et d'un autre côté dans la crédibilité de l'expert appuyée par des instances 
publiques qui ont, d'une certaine manière et avec bien des limites, validé ses compétences à travers la procédure d'agrément : le ministère du Travail, l'INRS et l'ANACT. ergonomie, tant du point de vue des acteurs qui en sont destinataires, de l'attention à la demande sociale (Teiger, 2006), que de celui des modalités d'intervention (Dugué, 2016). Une des particularités est que les transformations se situent davantage dans le champ des relations sociales que dans celui de transformations matérielles concrètes. Cette forme d'intervention peut être vue comme moyen de développer une culture SSCT dans les entreprises, mais aussi de rééquilibrer un peu le rapport de force au sein de l'entreprise entre les représentants des salariés et l'employeur (qui est aussi, rappelons-le, la fonction $\mathrm{du}$ Code $\mathrm{du}$ travail). Autrement dit, l'intervention ergonomique en expertise s'inscrit dans les fondamentaux (nous pourrions dire les valeurs) de la discipline tels que résumés par Dul, Bruder, Buckle, Carayon, Falzon et al. (2012), à savoir une combinaison unique de trois caractéristiques: une approche prenant en compte la complexité des systèmes, une pratique orientée par la conception (puisqu'il s'agit d'agir sur des projets de transformation), et la volonté de tenir en même temps les enjeux de santé ou de bien-être et de performance.

\subsection{L'intelligence situationnelle de l'intervenant comme clé de voûte des transformations}

Les transformations sont possibles parce que l'intervenant ergonome adopte une/des posture(s) favorisant leur émergence et leur faisabilité, et qu'il ne "s'enferme " pas dans le registre unique de l'expertise. En effet, même si la posture d'expert présente des avantages (crédibilité, écoute, facilité pour convaincre...), elle présente aussi des inconvénients (implication limitée des acteurs qui attendent les solutions de cet « expert»), ce qui peut apparaître contradictoire avec les démarches participatives défendues par l'ergonomie et le fait de considérer, comme il est souvent dit, que les travailleurs sont « experts de leur propre travail ». L'expert peut aussi avoir la volonté d'utiliser son autorité pour servir des intérêts socio-politiques ou pour appuyer une stratégie syndicale, par exemple dans la perspective d'élections professionnelles. Il est ainsi toujours possible de se cantonner dans le registre unique de «l'expert », car c'est parfois ce dont les acteurs pensent avoir besoin, et c'est aussi ce qui est implicitement attendu par le dispositif législatif. Pour autant, il ne s'agit pas d'exclure totalement ce registre, mais bien de s'en servir éventuellement pour aider le CHSCT à se positionner avec plus de légitimité. Grâce à son expertise, l'ergonome aura peut-être plus de facilités à sortir de ce registre pour potentialiser la réussite de son intervention, en s'inscrivant avant tout dans la perspective de la transformation et de l'action. Il convient aussi de souligner que les enjeux didactiques et de capitalisation, pour permettre au CHSCT de développer ses capacités d'analyse et d'action, sont d'ailleurs bien présents dans l'évaluation ministérielle des experts.

Il nous semble que l'expertise CHSCT s'appuie ainsi sur l'intelligence situationnelle : intelligence puisqu'elle nécessite de «sentir» les choses (attentes, inquiétudes, objectifs implicites ou cachés...) et de s'adapter en permanence, de jongler entre les différents registres et ce, sur un délai très court, ce qui complexifie la construction sociale de l'intervention. Il nous apparaît alors qu'il n'existe pas de posture idéale pour favoriser la mise en place des transformations, mais plutôt un équilibre des différentes 
façons de transmettre (tantôt expert, guide, accompagnateur, conseiller, médiateur, investigateur...), en fonction des moments, des acteurs et des contextes. De fait, nous pouvons considérer le positionnement social non pas comme un état, mais comme une situation d'équilibre, donc provisoire par essence, qui évolue et fluctue au cours de l'intervention. Cette "intelligence situationnelle", se construisant avec et par l'expérience, suppose bon nombre de compétences comme celles d'une connaissance fine $\mathrm{du}$ fonctionnement des relations sociales, du syndicalisme et des pratiques des acteurs dans ce domaine (Ponge, \& Dugué, 2017).

\subsection{Les limites rencontrées d'un point de vue méthodologique}

Dans ces travaux, plusieurs limites des méthodologies employées, à la fois dans leur mise en œuvre et dans les résultats produits, peuvent être pointées :

- L'impossibilité d'accéder à l'ensemble des éléments et phases de l'intervention: nécessité de passer par la verbalisation des consultants sur leurs actions et de se confronter aux ajustements qu'ils effectuent en permanence pour s'adapter à la situation en vue des résultats qu'ils cherchent à obtenir. Mais comme tout opérateur, l'intervenant est limité dans ses possibilités de verbaliser sa stratégie sous-jacente d'intervention (Berthelette, 2002).

- La combinaison à la fois de l'opérationnalité de la conduite de projet et de l'analyse de cette conduite : le temps passé à évaluer ne doit ne pas se faire au détriment de l'action (Landry, 2008) bien qu'il soit souvent difficile de combiner en un seul document des informations permettant de savoir où en est l'intervention par rapport au dispositif prévu (étapes réalisées, en cours de réalisation et non débutées)

- La variabilité de pratique des intervenants (manière d'animer, de percevoir et de pratiquer la conduite de projet) qui rend, de fait, plus complexe la compréhension de ce qui fait la réussite de l'intervention. L'analyse alors s'est basée essentiellement sur celle du chef de file puisque c'est son empreinte qui influera le plus sur la conduite de l'intervention.

- Avec cette démarche d'évaluation la question du comment (sont produits les résultats?) est beaucoup plus explorée que celle du quoi (quels sont les résultats obtenus?) (Landry, 2008).

- Le journal de bord aurait pu servir à améliorer l'intervention et la construire, mais il n'a pas été mis en discussion avec les autres intervenants.

\section{Conclusion}

Nous avons voulu montrer que l'expertise CHSCT/CSE portant sur les sujets de santé, sécurité et conditions de travail, bien que se déroulant dans un cadre fortement contraint (mais c'est aussi le cas de bien d'autres types de pratique comme nous l'avons souligné), constitue une forme de pratique de l'ergonomie parmi d'autres. Nos résultats attestent qu'il est parfois possible de dépasser certaines contraintes légales grâce aux relations et à la pédagogie que l'expert intervenant va déployer et à la démarche mise en œuvre dans l'intervention. Nos résultats illustrent également le rôle fondamental de l'ergonome pour alimenter le débat social sur le travail, contribuer à transformer le regard que portent les membres de l'instance sur les sujets à traiter, et finalement développer leur capacité d'agir sur le travail en se confrontant au terrain pour étayer leurs analyses (Dugué, 2016). Il nous semble que, particulièrement dans ce cadre de 
pratique où l'accompagnement à la mise en place de solutions n'est que peu ou pas possible, apporter de la connaissance sur le fonctionnement de l'Humain au travail et sur l'activité réelle de travail ne suffit pas à induire des transformations. Au-delà des connaissances produites, c'est bien la mobilisation des différents acteurs et leur implication qui permet d'obtenir des résultats favorisant la réussite de l'expertise. La construction technique de l'intervention est dans ce cas au service de la construction sociale, en particulier de la création de relations de confiance et de crédibilité indispensables à la coconstruction de l'intervention ergonomique, puisque c'est nécessairement à travers les échanges que se construisent et se préparent les transformations (Villena, 2001).

De fait, nos résultats montrent que, comme pour d'autres types d'intervention (Daniellou, 1999), les transformations dans le cadre de l'expertise portent en grande partie sur les représentations, les acteurs et leurs modes d'interactions, sur leurs pratiques, mais aussi sur l'intervenant lui-même. Le terme de "transformation" s'entend alors surtout en termes de dialogue social et de rapports sociaux entre les acteurs de l'entreprise plus qu'en termes matériel, technique et organisationnel des conditions de travail. Plus ici encore qu'ailleurs, l'ergonome doit développer un positionnement conscient et réfléchi avec chacun des membres du CSE pour permettre et enrichir les débats sur le travail, mais aussi pour les faire s'interroger sur leurs propres pratiques de représentation des salariés. Ce qui va permettre d'ouvrir la voie à d'autres transformations possibles.

Nous pouvons reprendre ici les mots de Catherine Teiger (2006): «le travail des ergonomes qui conceptualisent l'activité de travail des autres comme "action située" (...), est lui-même "situé" dans le contexte technique, social et scientifique. Les modèles, les connaissances acquises et les pratiques doivent donc toujours être resituées pour conserver leur pertinence ou être transformées ». Nous avons ainsi voulu dresser quelques spécificités de l'ergonomie dans son approche et sa manière d'intervenir dans le cadre de l'expertise en SSCT. L'éclairage sur le travail de l'ergonome dans ce type d'intervention peut permettre à des professionnels, encore en formation ou pas, de mieux appréhender les enjeux politiques et sociaux fortement présents au cœur des relations professionnelles, et les compétences qui sont nécessaires. Cela questionne les connaissances à acquérir lors des formations initiales et les compétences à construire dans la pratique professionnelle, notamment sur leur capacité à exploiter leur environnement pour servir la qualité de l'intervention et les transformations qu'ils cherchent à obtenir. Même si nous sommes convaincus que bon nombre d'éléments sont transposables avec la mise en place des CSE et des CSSCT, il est probable que certaines pratiques se rigidifient, particulièrement du fait de la réduction des délais pour traiter les demandes, de l'introduction du cofinancement, de la disparité des fonctionnements et des moyens selon les accords d'entreprises. Ce travail de recherche pourrait alors servir aussi de point d'appui pour illustrer l'impact probable de la mise en place des CSE sur le travail des « experts » ergonomes. 


\section{BIBLIOGRAPHIE}

Adam, H., \& Barnier, L.-M. (2013). La santé n'a pas de prix. Voyage au cœur des Comités hygiène sécurité et conditions de travail. Paris : Syllepse.

Arborio, A-M., \& Fournier, P. (1999). L'enquête et ses méthodes : l'observation directe. Paris : Nathan. Autissier, D. (2009). L'intelligence de situation, savoir exploiter toutes les situations. Paris : Eyrolles. Bachelier, E. (2016). Les modalités d'appropriation des expertises CE et CHSCT par les représentants du personnel. Thèse de doctorat en philosophie, Université Aix-Marseille.

Baribeau, C. (2004). Le journal de bord du chercheur. Communication présentée dans le colloque L'instrumentation dans la collecte des données, Hors-série, ( $\left.\mathrm{N}^{\circ} 2\right), 98-112$.

Becker, H. (1985). Outsiders ; Études de sociologie de la déviance. Paris : Éditions Métailié. (Coll. Leçons de choses).

Bellemare, M., Marier, M., \& Allard, D. (2001). Le journal de bord : un outil pour l'intervention et la recherche en ergonomie. In Actes du congrès SELF-ACE 2001, Les transformations du travail, enjeux pour l'ergonomie, pp. 58-62, Montréal.

Béguin, P., \& Clot, Y. (2004). L'action située dans le développement de l'activité. Activités, 1(2), 35-49.

Berthelette, D. (2002). Les déterminants de l'implantation et des résultats des programmes de santé et de sécurité du travail. In D. Harisson, \& C. Legendre (Eds.), Santé et transformations du travail. Réflexions et recherches sur le risque professionnel (pp. 107-128.) Sainte-Foy : Les presses de l'Université du Québec à Montréal.

Boutte, J.-L. (2007). Transmission de savoir-faire. Réciprocité de la relation éducative Expert-Novice. Paris : L'Harmattan.

Corradi, A. (2015). Ergonomie et Instances Représentatives du Personnel : quel rôle, quelle coopération en vue de développer le pouvoir d'agir des salariés? Communication présentée au $50^{e}$ Congrès de la SELF, Paris.

Cristofalo, P. (2009). L'institutionnalisation d'une fonction d'expertise et de conseil auprès des élus du personnel. Cahiers internationaux de sociologie, 1(126), 81-98.

Daniellou, F. (1996). L'ergonomie en quête de ses principes. Toulouse : Octarès.

Daniellou, F. (1999). Le statut de la pratique et des connaissances dans l'intervention ergonomique de conception. Collection Thèses et Mémoires, Laboratoire d'Ergonomie des Systèmes Complexes, Université Bordeaux 2.

Daniellou, F. (2006). Entre expérimentation réglée et expérience vécue : Les dimensions subjectives de l'activité de l'ergonome en intervention. Activités, 3(1), 5-18.

Delmas, C. (2011). Sociologie politique de l'expertise. Paris : La Découverte (collection Repères).

Dugué, B. (2016). Expertise CHSCT : des débats toujours d'actualité. In M. Christol-Souviron, S. Leduc, A. Drouin, \& P. Etienne (Eds.), «Performances Humaines \& Techniques », d'hier vers aujourd'hui (pp. 619-627). Toulouse : Éditions Octarès.

Dugué, B., \& Petit, J. (2018). La discussion des modèles d'action, une condition pour l'action des comités en santé et en sécurité au travail. Relations Industrielles/Industrial Relations, 73(2), 252-273. 
Dugué, B., Petit, J., \& Daniellou, F. (2010). L'intervention ergonomique comme acte pédagogique. Perspectives interdisciplinaires sur le travail et la santé. [En ligne], mis en ligne le 1er novembre 2010, consulté le 19 mars 2017. URL : http://pistes.revues.oirg/2767 ; DOI : 10.4000/pistes.2767

Dugué, B., Petit, J., \& Pinatel, C. (2012). Les CHSCT : entre dispositifs et pratiques. Lyon, France : Réseau ANACT.

Dul, J., Bruder, R., Buckle, P., Carayon, P., Falzon, P., Marras, W., Wilson, J.R., \& van der Doelen, B. (2012). A strategy for human factors/ergonomics: developing the discipline and profession. Ergonomics, 55(4), 377-395.

Etienne, P., \& Négroni, P. (1999). Du droit à l'expertise à une évolution des pratiques. Performances Humaines et Techniques, 102, 23-27.

Goffman, E. (1959). La mise en scène de la vie quotidienne. Paris : Les éditions de Minuit.

Goffman, E. (1974). Les rites d'interaction. Paris : Les éditions de Minuit.

Guérin, F., Laville, A., Daniellou, F., Duraffourg, J., \& Kerguelen, A. (1997). Comprendre le travail pour le transformer, la pratique de l'ergonomie. Lyon : ANACT.

Guillon, F. (2002). La pratique de l'ergonomie quand tout pousse à l'expertise... Communication présentée au $37^{e}$ Congrès de la SELF, Aix-en-Provence, 145-154.

Hammond, K., Converse, T., \& Grass, J. (1992). The Stabilization of Environments. Artificial Intelligence, 72(1-2), 305-327.

Hughes, E. (1996). Le regard sociologique. Paris : École des Hautes Études en sciences sociales.

Kirsh, D. (1995). The intelligent use of space. Artificial Intelligence, 73(1-2), 31-68.

Lameul, G. (2008). Les effets de l'usage des technologies d'information et de communication en formation d'enseignants, sur la construction des postures professionnelles. Savoirs, 17, 71-94.

Landry, A. (2008). L'évaluation de l'intervention ergonomique : de la recherche évaluative à la proposition d'outils pour la pratique. Thèse de doctorat en ergonomie, Université Bordeaux 2.

Landry, A., \& Tran Van, A. (2010). L'évaluation, un outil de l'ergonome pour transformer le contexte d'intervention. Perspectives interdisciplinaires sur le travail et la santé. [En ligne], mis en ligne le 1er mai 2010, consulté le 20 mars 2017. URL : https://pistes.revues.org/2473 ; DOI : $10.4000 /$ pistes. 2473

Leplat, J., \& Cuny, X. (1974). Les accidents du travail. Paris : PUF.

Litim M., \& Castejon, C. (2010). Protéger la santé des travailleurs : pour que la mission du CHSCT ne devienne pas impossible. Nouvelle revue de psychosociologie, 2010/2(10), 139-150.

Litim, M., Zittoun, M., \& Briec, C. (2012). L'intervention au-delà de l'expertise CHSCT : entre action et instrument d'action. Bulletin de psychologie, 2012/3 (519), 227-237.

Nivelet, N., \& Chevillard, F. (2019). Comité social et économique. Prérogatives en santé, sécurité et conditions de travail. Paris : INRS, ED 6340.

Petit, J., Querelle, L., \& Daniellou, F. (2007). Quelles données pour la recherche sur la pratique de l'ergonome? Le Travail Humain, 70(4), 391-411.

Ponge, L., \& Dugué, B. (2017). L'enjeu de la prévention primaire lors des projets de changement : quelle place pour le CHSCT ? Quels apports de l'expertise ? Activités, [En ligne], mis en ligne le 15 avril 2017, consulté le 18 avril 2017. URL : https://journals.openedition.org/activites/2959 
Rabardel, P., Carlin, N., Chesnais, M., Lang, N., Le Joliff, G., \& Pascal, M. (1998). Ergonomie, concepts et méthodes. Toulouse : Octarès.

Samurçay, R., \& Rabardel, P. (2004). Modèles pour l'analyse de l'activité et des compétences : propositions. In R. Samurçay \& P. Pastré (Eds.), Recherches en didactique professionnelle

(pp. 163-180). Toulouse : Octarès.

Such, F. (2017) Comment faire pour que les interventions en santé et sécurité au travail aient des effets durables dans l'entreprise. Communication présentée au $52^{e}$ Congrès de la SELF, Présent et Futur de l'Ergonomie, Toulouse, pp. 145-149.

Suchman, L. (1987). Plans and situated actions : the problem of human machine interaction. Cambridge : Cambridge University Press.

Teiger, C. (2006). Quand les ergonomes sont sortis du laboratoire... À propos du travail des femmes dans l'industrie électronique (1963-1973). PISTES, 8(2), 38 p.

Teiger, C. (2008). Entrevue guidée avec Hélène David et Esther Cloutier. PISTES, 10(1), 21 p.

Tran Van, A. (2010). Pratique collective de l'intervention ergonomique : comment les ergonomes font-ils pour travailler ensemble? L'exemple de la pratique des ergonomes consultants. Thèse de doctorat en ergonomie, Bordeaux, Université Bordeaux 2.

Villena, J. (2001). L'argumentation en ergonomie : l'accompagnement à la conduite de projet et la construction de solutions. Communication présentée aux Journées de Bordeaux sur la pratique de l'ergonomie «L'ergonome et les solutions », Bordeaux, pp. 17-25.

\section{NOTES}

1. Dans la suite de cet article, dans un souci de simplification de l'écriture, chaque fois que le terme CHSCT sera évoqué, la notion de CSE sera sous-entendue au vu de la similitude de leurs prérogatives d'un point de vue HSCT. Quand nous parlerons « d'expertise CHSCT », il s'agira donc d'évoquer l'expertise en santé, sécurité et conditions de travail (SSCT) à laquelle le CSE peut avoir recours.

2. Le CHSCT est une instance représentative du personnel regroupant à la fois l'employeur et les représentants du personnel. Des invités permanents et, de manière exceptionnelle des membres extérieurs, peuvent y intervenir en tant que spécialistes et exercer ainsi une fonction de conseil auprès des membres de la direction et des élus. Des CHSCT existent encore dans la Fonction Publique.

3. Le cadre législatif prévoit de faire évoluer le principe d'agrément des experts vers une notion d'habilitation d'ici mars 2022 (R. 4614-6 à R. 4614-17).

\section{RÉSUMÉS}

L'expertise CHSCT - désormais expertise pour les CSE en santé, sécurité et conditions de travail est une forme de pratique de l'ergonomie. Si ce n'est pas la seule discipline à intervenir dans ce cadre, nous supposons néanmoins qu'elle y répond avec certaines spécificités contribuant à la 
mise en œuvre de transformations. En se basant sur l'étude de plusieurs cas d'intervention dans le cadre d'expertises "projet important ", nos analyses démontrent que les transformations sont possibles et multiples : portant sur les relations sociales, les représentations des acteurs, leurs modes d'interactions, mais aussi sur l'intervenant. La posture et l'intelligence situationnelle de ce dernier sont d'ailleurs des éléments clés de la réussite d'une expertise. Ainsi, si ce mode d'intervention est souvent source de critiques en ergonomie, cela vient sans doute de la nature de ses transformations, moins visibles, qui ne sont pas forcément techniques, matérielles ou organisationnelles, mais avant tout profondément sociales.

HSWC expertise- now CSE expertise (social and economic committees in French companies) in Health, Safety and Working Conditions- is a form of ergonomics practice. Ergonomics is not the only discipline to intervene in this context but we assume that it responds with specificities contributing to the implementation of transformations. Our analyses, based on the study of several cases of intervention in the context of "important project" expertise, demonstrate that transformations are possible and varied with regard to : social relations, the representations of actors, their modes of interaction and the ergonomists themselves. The latter's position is also a key element in the success of an expertise. So if this mode of intervention is often a source of criticism in ergonomics, it is undoubtedly due to the nature of its less visible transformations, which are not necessarily technical, material or organizational but above all deeply social.

\section{INDEX}

Keywords : HSWC expertise, ergonomic intervention, transformations, practitioner's posture, situational intelligence

Mots-clés : expertise SSCT, intervention ergonomique, transformations, posture de l'intervenant, intelligence situationnelle

\section{AUTEURS}

\section{CLAIRE VIOLLEAU}

Équipe Ergonomie des Systèmes Complexes IMS, Bordeaux-INP. Avenue des Facultés, 33400 Talence. violleau.claire@gmail.com

\section{BERNARD DUGUÉ}

Équipe Ergonomie des Systèmes Complexes IMS, Bordeaux-INP. Avenue des Facultés, 33400

Talence. bernard.dugue@bordeaux-inp.fr 